\title{
A Virtual Wisdom Mining 'Pan' For Connecting Retired Experts With Currently Active Professionals
}

\author{
Nirmalya Thakur \\ University of Cincinnati \\ Cincinnati, OH 45221-0030, USA \\ thakurna@mail.uc.edu.edu

\section{Chia Y. Han} \\ University of Cincinnati \\ Cincinnati, OH 45221-0030, USA \\ han@ucmail.uc.edu
}

2018 IT Research Symposium, April 10, 2018, Cincinnati, Ohio, USA. Copyright is held by the owner/author(s). Publication rights licensed to the University of Cincinnati. The IT Research Symposium reserves the right to provide a hyperlink to the author's site if the Material is used in electronic media.

\begin{abstract}
With the average life span increasing these days, there has reportedly been an increase in population of the retired professionals and the further aged people. Before retirement these people had been pioneers in their field but post retirement their knowledge, experience and wisdom go under-utilized. While these retired people have less chances to contribute to the technological development, it becomes difficult for the new industry recruits to easily fill in the shoes of these retired experts at the industry level. In view of the above problem, this paper proposes an application named 'Wisdom Pan' which is a virtual wisdom mining pan for reducing the gap between retired industry professionals and current industry workers. With the help of this application, retired professionals can sit at the comfort of their homes and share their knowledge and expertize as stories which can help the current industry workers.
\end{abstract}

\section{Author Keywords}

Knowledge capture; wisdom-mining; virtual booth story-telling; multiple-language, Android app; usability testing; universal diversity 


\section{Introduction}

A person's life cycle goes through several distinct phases, in terms of needs and physical conditions $[1,2]$. It can be roughly described in eight major stages: (1) child (age 1-12), (2) youth (age 12-18), (3) young adult (19-22), (4) young family (age 23-28), (5) career adult (age 29-45), (6) senior career adult (age 46-65), (7)retired adult (age 66-85), and (8) elderly (age 80 + ). Each group has its own distinct needs and life styles. People live longer nowadays. This means that the overall population of the last three groups is not dwindling as fast as it used to [3].

With the transition of each group to the next, a major change of life style takes place. Different life experiences are gained by each person, based on the context of many varied factors, such as culture, place, and time. The focus of this work is to connect the Group 6 and Group 7.

Currently they are represented by the so-called Generation-X, the 'boomer generation' after the WWII, the hippy generation of the 60's and 70's [4]. These people have been the backbone of the working society and have built a solid financial nest. More importantly, due to the fast growth of science and technology, these people have accumulated a wealth of experience in keeping up with the fast pace of knowledge growth, especially, within a given trade or industry sector. Many of them are pioneers in their field, and many are the users of the many generations of the technological systems. They have a very good grasp of the technological know-how and original ideas for the solutions to the needs of the society.
When they leave the working force, they carry with them all the wisdom. It is desirable that this wisdom can be captured and saved for the future generations this forms the main motivation for developing this application.

Another motivation for developing this application is the user diversity in terms of language [5]. The Ethnologue catalogue of world languages [6], which is one of the best linguistic resources, currently lists 6909 living languages. About $6 \%$ of them have more than a million speakers each, and collectively account for $94 \%$ of the world population. So, people in different parts of the world could face difficulty to connect if they are speaking different languages. Thus, this application also seeks to address this issue by allowing the storage of responses in sixteen international languages which are amongst the most widely spoken languages in the world $[7,8]$

\section{Related Work}

A great deal of studies has been conducted to understand how retirement affects the life of a person [9] and how retired people can stay connected to technologies [10]. Different research works have also been aimed at involving the retired people in different domains of work with the other generations. Mao Mao et al. [11] proposed a technique to keep retired people involved with the community by connecting them through music. Benoit et al. proposed an activity management system to empower the social lives of retired people by helping them to connect to social events [12]. Ricardo et al. conducted a study to analyze the effect of retirement on the psychological well-being of a person and proposed methods to educate them so that they can stay connected in terms 


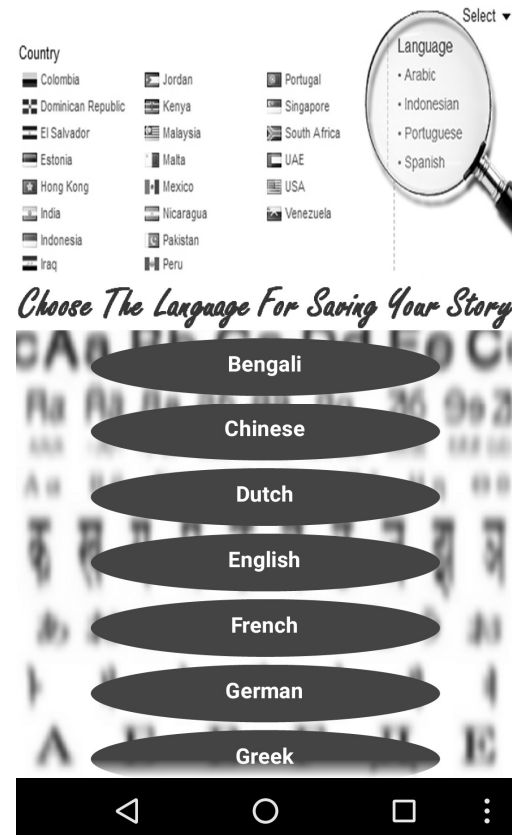

Figure 1: Application screenshot showing some of the languages in which the user-stories can be stored. of technological developments [9]. Although similar studies $[13,14]$ have been conducted on studying the lives of retired people and helping them to stay connected, very few works have been aimed at capturing the knowledge and wisdom of the retired people for helping the current industry professionals.

This work thus presents an application 'Wisdom Pan' which is aimed at making the retired people share their experience and expertize as stories, thereby helping the current generations who are required to fill in their shoes. The fact that this application also incorporates the translation of the story into some of the most widely spoken languages of the world $[7,8]$, upholds the universal usability of this application. Since this application serves as a tool for collecting stories containing wisdom 'nuggets', just like in old gold rush days in wild west, the tool for getting the gold was through gold panning utensils; this application is effectively called 'Wisdom Pan'.

\section{Proposed Work}

This 'Wisdom Pan' App is a multilingual virtual 'storytelling booth' for Generation-X people for sharing their knowledge through stories, such that people can pan for their wisdom. This application allows them to share their experience and knowledge in the form of stories and is aimed at connecting them to the professionals who are still working. As the world is now 'flat' in the sense that people everywhere in the world are connected through Internet, the application also allows the users to store the stories in some of the most widely spoken languages of the world $[7,8]$ to address Universal Diversity in terms of language. This is illustrated in Figure 1.
The users of this application can thus be broadly divided into two groups - Group I being the

Generation-X people, who can share their knowledge and wisdom though the application. Group II being the current industry workers who can refer to these stories shared by the retired people. Thus, according to the user groups, this android application presents the user with two broad options - either to share a story or to read the existing stories.

Once the user chooses to share a story the speech recognizer component [15] recognizes the user's spoken words which are then analyzed by the speech to

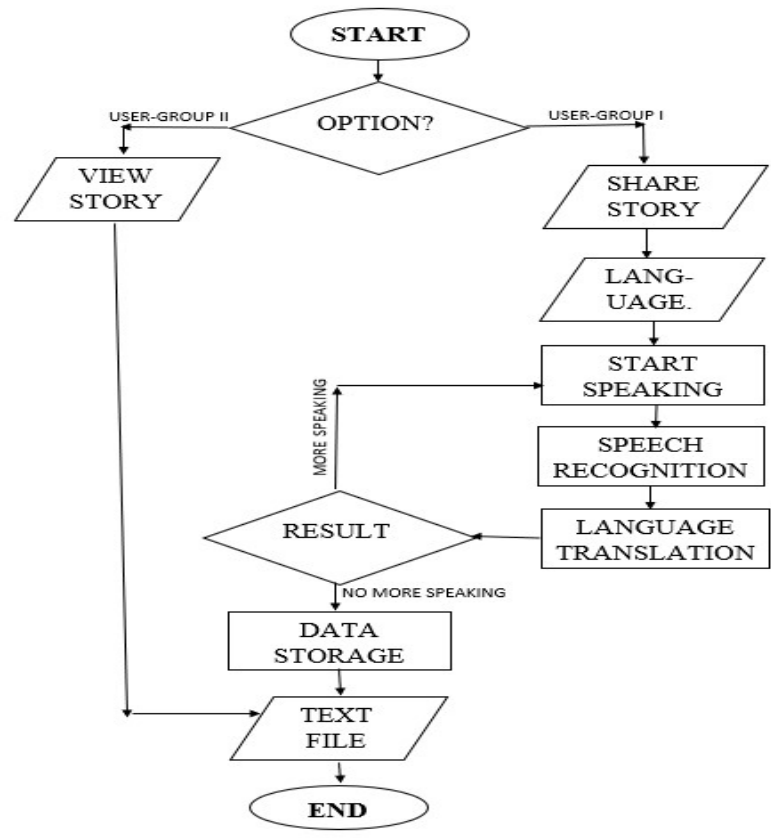

Figure 2: Flowchart of the Application 


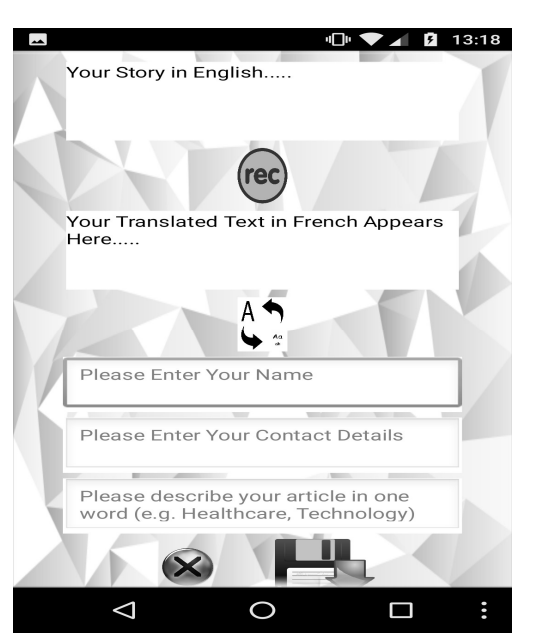

Figure 3: A typical screenshot from the application when the user chooses to save the shared story in French

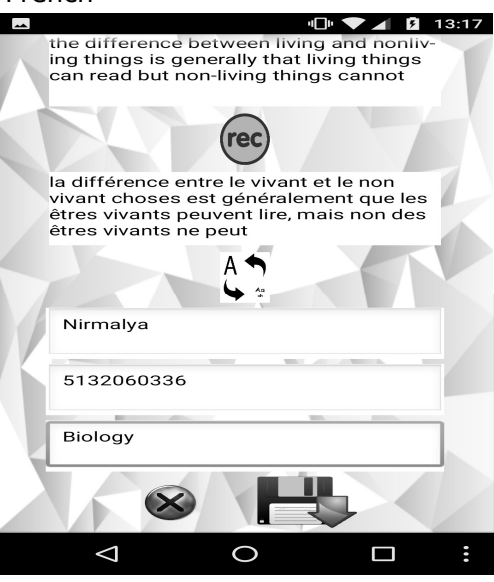

Figure 4: A typical screenshot from the application when the user shares a story in French text converter [16] which converts the speech to text. This conversion is aimed to remove any noise or disturbances in the recorded audio and to ensure that the response can be well perceived by the other user group irrespective of the accent, pronunciations or other speech modulations of the user who has shared the response. After the conversion, the language translator [17] component of the application converts the captured words into the language the user wishes the response to be translated to. During this phase of speech recognition and translation, the application implements effective user feedback and actionresponse compatibility by displaying to the user the captured words in the audio and the translated text. The user also gets ample warm-up time as the user can perform any number of trials before deciding to save the response. This fact is illustrated in the flowchart of the application in Figure 1.

Thereafter, prior to saving, the user is prompted to provide his or her name and contact information which gets added to the end of the user's story automatically, so that the other user group can have further communications if they are interested in knowing more on that topic from the person who shared that response.

Finally, before saving the user is also asked to describe the domain of the response in one word, (for example healthcare, technology etc.) which allows better sorting of the individual text files in which the respective stories get saved, as the domain name gets added to the name of the text file each time, during the process of saving the response. Some of these features are illustrated in Figure 3 and Figure 4
It is worth mentioning here that each response shared by a user gets stored as a separate file in the system and all the stored files are available for future read at any point of time.

The text file name contains the topic of the talk, expressed as one word, followed by the language in which it is saved, and it also features the author's name. This not only allows sorting of the different responses in the system as per the topics of the stories but also ensures an enhanced user experience by ensuring that the user who is keen to read the stories gets to know the topic, the language and the name of the person who has shared the story by just reading the filename. For instance, as per Figure 4, the story shared by the user would appear as

'Biology_French_by_Nirmalya.txt' in the system.

\section{Sample Run and Results}

During the Usability Testing phase 11 volunteers (7 males and 4 females) between the ages 19-55 participated in the testing. Prior to the Usability Testing the volunteers were briefed about the application to make them aware of its purpose and functionalities and the testing involved volunteers participating as users belonging to the Group I category as well as users belonging to the Group II category. While the older users were asked to work as Group I users and give their feedback while sharing a story, the younger users were asked to work as Group II users and look for a story which had already been shared by Group I users, their feedback was also recorded in this regard.

The Usability Testing involved recording the users feedback on the four broad parameters of design, functionality, effectiveness and performance while they 

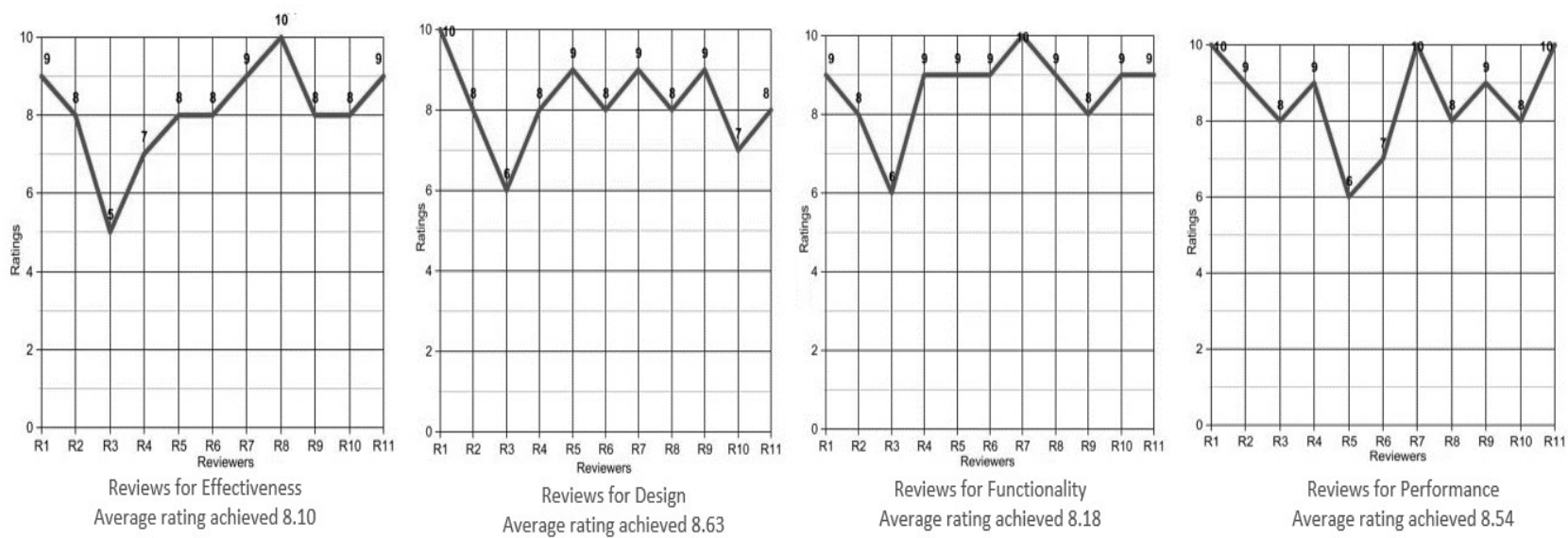

Figure 5: Results of Usability Testing as user-surveys performed by 11 volunteers ( 7 males and 4 females) between the ages $19-55$.

used the application. The user feedback was recorded in the form of user-surveys which involved the users to give their views and feedback on different aspects of the application primarily concerning these four major aspects through questionnaires. The results of Usability Testing were considerably appreciable and as shown in Figure 5, the application received an average rating of more than 8 on a scale of 1 to 10 , in the different parameters which were being evaluated during the Testing.

\section{Acknowledgements}

We thank all the volunteers who not only participated in the Usability Testing but also gave their valuable feedback by participating in the user-surveys.

\section{Conclusion and Future Work}

In this paper an android application - 'Wisdom Pan' has been proposed for connecting Generation-X people with currently active professionals.
This application allows Generation-X people to share their knowledge, expertize and wisdom conveniently in the form of stories which are stored for current and future reference of the industry professionals. This application also aims at addressing Universal Diversity through its multilingual feature - which allows storing of the responses in some of the most widely spoken languages of the world $[7,8]$. As per the best knowledge of the authors, there hasn't been any other study conducted in this area featuring a similar approach.

The results of Usability Testing prove that this application not only meets the approached problem statement but also meets the user expectations and features a great user experience thereby connecting retired professionals to currently active professionals. Future work on this application would involve implementing the same for IOS, Windows and other platforms to ensure that the application can be run on 
any system or device irrespective of its operating system, thus enhancing user experiences.

\section{References}

1. David Elkind, Dr. C. George Boeree "Personality Theories", Psychology Department Shippensburg University, Published 1997.

2. Asghar Zaidi, "Life Cycle Transitions and Vulnerabilities in Old Age: A Review", 2014 UNDP Human Development Report Office Occasional Paper.

3. Birkland, J. L. H., \& Kaarst-Brown, M. L. (2010). 'What's so special about studying old people?': The ethical, methodological, and sampling issues surrounding the study of older adults and ICTs. In F. Sudweeks, H. Hrachovec \& C. Ess (Eds.), Proceedings of the seventh international conference on Cultural Attitudes Towards Technology and Communication (pp. 341-356). Vancouver, B.C., Canada.

4. Jeni McIntosh-Elkins, Karen McRitchie, Maureen Scoones "From the Silent Generation to Generation $X, Y$ and $Z$ : Strategies for Managing the Generation Mix" SIGUCCS'07, October 7-10, 2007, Orlando, Florida, USA.

5. W. Tecumseh Fitch "Unity and diversity in human language "Philos Trans R Soc Lond B Biol Sci. 2011 Feb $12 ; 366(1563)$ : 376-388. doi: $10.1098 /$ rstb. 2010.0223 .

6. Ethnologue catalogue of world languages, Available https://www.ethnologue.com.

7. List of languages by number of native speakers, Wikipedia the free encyclopedia, Available https://en.wikipedia.org/wiki/List_of_languages_by _number_of_native_speakers.

8. Paolillo, John C.; Das, Anupam (31 March 2006). "Evaluating language statistics: the
Ethnologue and beyond" (PDF). UNESCO Institute of Statistics. pp. 3-5. Retrieved 8 October 2015.

9. Ricardo Pocinho, Esperanza Navarro-Pardo, Juan José Fernández-Muñoz "Study of the Perception of Active Workers in Preparation for Retirement: Analysis of the Effect of the Education for Retirement in Psychological Well-being" TEEM'16, November 02-04, 2016, Salamanca, Spain.

10. Johanna L.H. Birkland "A Mixed Bag: How Work and Retirement Influence Older Adults' ICT Use" SIGMIS-CPR'12, May 31-June 2, 2012, Milwaukee, Wisconsin, USA.

11. Mao Mao, Johanna M. Lukate, Alan F. Blackwell, David A. Good "Supporting Retirement Socially and Musically by Technology: An Ethnographic Study of Local Community Musicians" CHI'16 Extended Abstracts, May 07-12, 2016, San Jose, CA, USA

12. Benoît Otjacques, Marc Krier, Fernand Feltz, Dieter Ferring, Martine Hoffmann "Helping older people to manage their social activities at the retirement home" HCI 2009 - People and Computers XXIII, Published by the British Computer Society.

13. Keith A. Bender and Natalia A. Jivan "what makes retirees happy?" An issue in brief center for at Boston college research retirement February 2005, number 28.

14. Lola M. Lrelan "Retirement History Study: Introduction" Division of Retirement and Survivor Studies, Office of Research and Statistics" Bulletin, November 1972.

15. Google Speech Recognizer Application, Available https://cloud.google.com/speech/.

16. Google Speech to Text Application, Available https://cloud.google.com/speech/docs/.

17. Yandex Translator Application, Available https://tech.yandex.com/translate/. 